\title{
Quels traitements et interventions sont-ils encore autorisés?
}

\author{
Nils Graf \\ Lic. en droit, service juridique FMH
}

L’article 10a, al. 2, de l'ordonnance 2 Covid-19, entré en vigueur le 21 mars 2020, interdit aux établissements de santé, notamment aux hôpitaux, aux cliniques, aux cabinets médicaux et aux cabinets dentaires, de réaliser des examens, des traitements et des thérapies (interventions) non urgents. Cette mesure se répercute de manière très sévère sur l'activité de nombreux médecins indépendants, qui ne voient plus actuellement que $10 \%$ de leur patientèle. Ils doivent toutefois continuer de couvrir de nombreux coûts comme les loyers ou la location d'équipements et appareils médicaux. C'est pourquoi nous avons tenu à dresser ci-après un inventaire des activités et des gestes restant autorisés et que les médecins peuvent continuer d'effectuer durant la présente période de crise.

Nous soulignerons tout d'abord qu'il relève en dernier lieu de la compétence des professionnels de santé de décider si une intervention est nécessaire ou non. Le jugement du médecin est l'élément décisif. Les médecins sont invités à user de cette marge d'appréciation et à prendre leurs décisions en fonction de chaque cas individuel.

L'article 10a, al. 3, de l'ordonnance 2 Covid-19 (état au $1^{\text {er }}$ avril 2020) précise ce qu'il faut entendre par «non urgent». Sont notamment considérées comme non urgentes les interventions:

a. qui peuvent être réalisées à une date ultérieure sans que la personne concernée ne risque de subir d'autres inconvénients que des atteintes ou des troubles physiques et psychiques mineurs, ou

b. qui sont réalisées, principalement ou entièrement, à des fins esthétiques ou pour améliorer les performances ou le bien-être.

Ces dispositions visent deux objectifs, qui peuvent servir (cumulativement) de lignes directrices pour les traitements devant continuer d'être administrés:

D'une part, il convient d'éviter des rassemblements inutiles dans ces établissements (p. ex. dans les salles d'attente) et de faire en sorte que seules y soient présentes les personnes ayant immédiatement besoin d'un traitement. D'autre part, les capacités et les ressources potentiellement nécessaires au traitement des patients infectés par le Covid-19 (ressources en personnel, infrastructures, médicaments et consommables) ne doivent pas être mobilisées par des interventions qui ne sont pas indispensables d'un point de vue médical. Cela exige de tous les acteurs du domaine de la santé qu'ils prennent aujourd'hui leurs responsabilités et fixent des priorités pour les mois à venir. En particulier, il est urgent de limiter le nombre de séjours hospitaliers inutiles dans les établissements de soins intensifs et intermédiaires. Par conséquent, seuls les interventions chirurgicales et les traitements considérés comme vitaux doivent être effectués. Les décisions prises doivent néanmoins garantir l'accès à des soins de qualité au plus grand nombre de patients possible.

Inversement, cela signifie également que les médecins sont tenus de traiter tous les problèmes de santé qui, s'ils n'étaient pas traités, pourraient entraîner une éventuelle hospitalisation ultérieure. A titre d'exemple, on peut citer une infection de l'oreille moyenne. Si elle n'est pas traitée, elle risque de se péjorer au point que le patient sera contraint de se rendre au service des urgences d'un hôpital un jour ou deux plus tard.

Conformément au rapport explicatif concernant l'ordonnance 2 Covid-19, «sont entre autres autorisées les interventions qui, si elles ne sont pas effectuées, entraînent une réduction de l'espérance de vie, une lésion irréversible, un risque considérable d'aggravation de l'état de santé ou une hospitalisation d'urgence, ou qui dégradent la qualité de vie de façon extraordinaire. Exemples d'interventions autorisées en particulier:

- chirurgie tumorale dans toutes les disciplines lorsque la tumeur est nocive ou mortelle,

- opérations vasculaires qui, si elles ne sont pas effectuées, entraînent la perte permanente de la fonction d'un membre,

- hernies irréductibles ou incarcérées de tous types,

- opérations des articulations qui, si elles ne sont pas effectuées, entraînent une restriction permanente de la fonction,

- fractures qui ne peuvent pas être traitées de manière conservatrice, 
- opérations du dos en cas de défaillance ou de douleur incontrôlable,

- toutes les interventions liées à la grossesse et à l'accouchement,

- les états de douleur aiguë qui nécessitent un traitement opératoire,

- interventions pour les états infectieux qui ne peuvent pas être maîtrisés de manière conservatrice (p. ex. les abcès),

- rétablissement de la capacité de fonctionnement des professionnels médicaux,

- prestations de télémédecine ainsi que

- interventions préventives chez les enfants et les adolescents (vaccins).»

Il n'est pas possible d'établir une liste exhaustive de toutes les interventions médicales autorisées. Dans le secteur ambulatoire, on autorise toutes les consultations ayant pour but de déterminer si un problème est vital, particulièrement dans le domaine de la pédiatrie. Les contrôles de traitements prodigués avant l'entrée en vigueur de l'ordonnance doivent bien entendu être effectués, dans la mesure où il existe une indication médicale en ce sens. On veillera bien sûr aussi à ce que le cabinet respecte les consignes d'hygiène de l'OFSP. Il importe par ailleurs d'échelonner l'arrivée des patients au cabinet de manière que le plus petit nombre possible de personnes séjourne dans les salles d'attente à chaque moment donné.

Les cabinets et cliniques de chirurgie esthétique n'ont en aucun cas le droit de continuer à consulter ou d'effectuer des traitements tels que des injections de collagène, etc. Les cabinets dispensant des soins médicaux mais également des traitements visant à améliorer le bien-être ou les performances ne sont pas autorisés à proposer ce type de prestations.

Pour conclure, on fera remarquer qu'il appartient toujours au professionnel de la santé de décider au cas par cas si une intervention est oui ou non nécessaire. Pour parer aux éventuelles remises en question ultérieures de ces décisions, le médecin veillera à documenter scrupuleusement et de manière claire les raisons qui l'ont amené à déterminer qu'une intervention ou un

traitement ne pouvaient être reportés.

chen vigueur delordonnance doivent bien entendu être

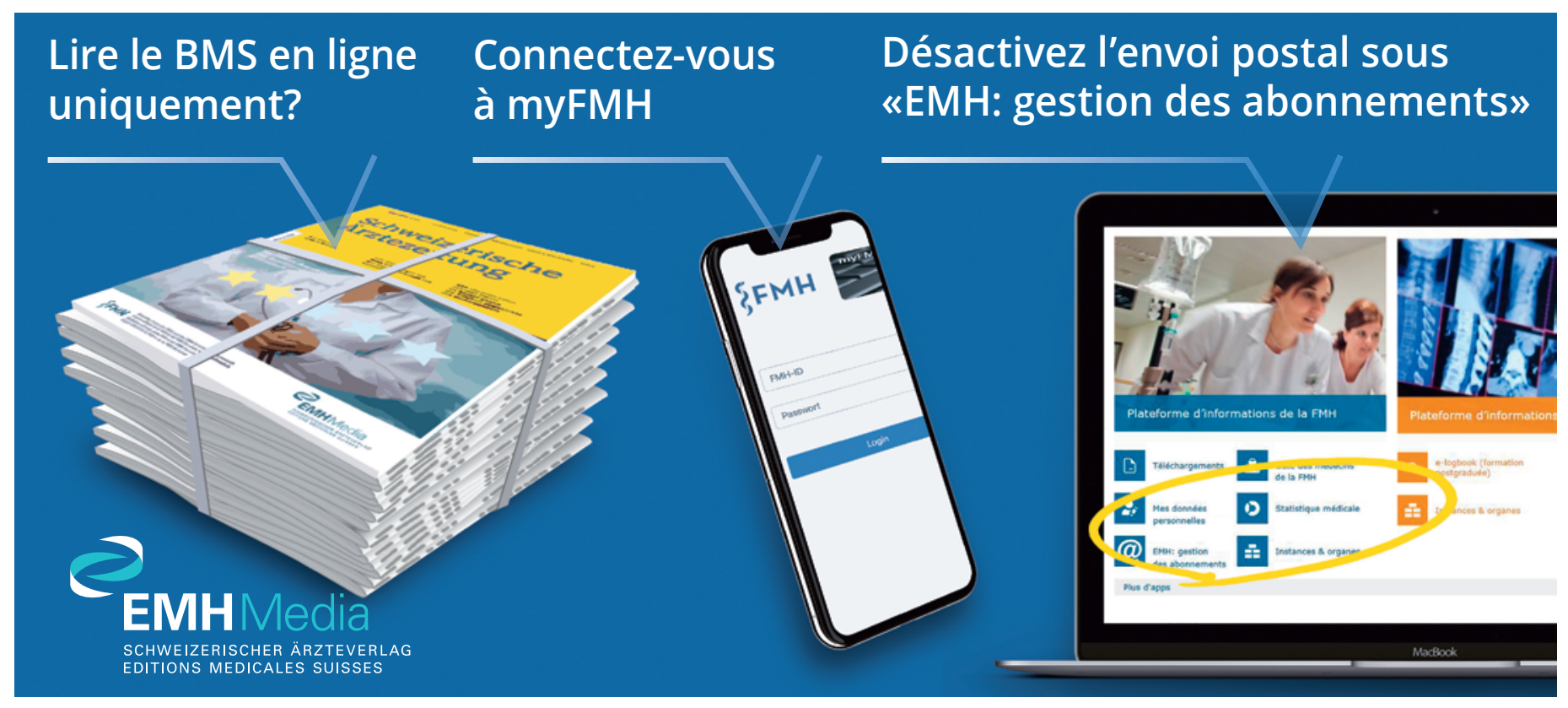

